\title{
Técnicas quirúrgicas de exclusión de la orejuela auricular izquierda: revisión a propósito de un caso
}

\begin{abstract}
Cristina Margarita Lanfranco
Guillermo Godoy-Zammarrelli

Fabricio Massaccesi

María Laura Plastino
\end{abstract}

Vanessa Marasa

\section{Correspondencia}

Cristina Margarita Lanfranco cris.lanfranco@gmail.com

Unidad de Eco Doppler Cardiovascular. Área de Cardiología. Hospital Italiano de La Plata. Buenos Aires. Argentina

Recibido: 14/01/2020

Aceptado: 14/01/2020

En línea: 02/03/2020

Citar como: Lanfranco CM, Godoy-Zammarrelli G, Marasa V, Massaccesi F, Plastino ML. Técnicas quirúrgicas de exclusión de la orejuela auricular izquierda: revisión a propósito de un caso. Rev Ecocar Pract (RETIC). 2020 (Mar); 3 (1): 1-6. doi: 10.37615/retic.v3n1a2.

Cite this as: Lanfranco CM, Godoy-Zammarrelli G, Marasa V, Massaccesi F, Plastino ML. Surgical techniques of the left atrial appendage exclusion: a case based review. Rev Ecocar Pract (RETIC). 2020 (Mar); 3 (1): 1-6. doi: 10.37615/retic.v3n1a2.

\section{Palabras clave \\ $\triangleright$ Orejuela auricular izquierda \\ $\triangleright$ Fibrilación auricular \\ $\triangleright$ Accidente cerebrovascular}

\begin{abstract}
RESUMEN
La orejuela auricular izquierda es la fuente embolígena más frecuentemente hallada en pacientes con fibrilación auricular y accidente cerebrovascular. En la etapa postoperatoria de la cirugía cardíaca la prevalencia de fibrilación auricular es elevada. La exclusión de la orejuela ha surgido como un método potencial para reducir los eventos isquémicos en el postoperatorio de cirugía cardíaca. Se han desarrollado y empleado diferentes técnicas quirúrgicas, con tasas de éxito variables, sin evidencia definitiva que avale su indicación de rutina para la reducir la tasa de accidente cerebrovascular en pacientes con fibrilación auricular sometidos a cirugía cardíaca.
\end{abstract}

\section{Introducción}

La fibrilación auricular (FA) es la arritmia más frecuente en la población general y un factor de riesgo independiente de morbimortalidad cardiovascular. A su vez, la prevalencia de FA está en aumento, coincidente con el envejecimiento de la población mundial(1)

Es bien conocido que la FA es un factor de riesgo definido para la ocurrencia de accidente cerebrovascular (ACV) isquémico relacionado con la presencia de trombos en la orejuela auricular izquierda $(\mathrm{OAI})^{(2-3)}$. En los países occidentales, el ACV es la tercera causa de muerte y la primera causa de discapacidad, siendo la etiología isquémica la más prevalente.

La OAI presenta una morfología que, en presencia de FA, es propensa al estasis sanguíneo y formación de trombo. La terapia anticoagulante es el tratamiento médico más efectivo en la prevención del ACV relacionado con la existencia de FA. Sin embargo, el riesgo de sangrado y de ACV hemorrágico restringe el uso indiscriminado de fármacos anticoagulantes. El cierre de la orejuela ofrece una estrategia potencial para la prevención del ACV relacionado con la formación de trombos en la OAl, como tratamiento alternativo o adyuvante a la terapia anticoagulante, en pacientes con ritmo de FA que son sometidos a cirugía cardíaca (valvular, cirugía de revascularización miocárdica, etc.)

En este artículo se realiza una revisión de los resultados publicados sobre las técnicas quirúrgicas de oclusión de la OAl, a propósito de un caso clínico en el que se efectuó la exclusión quirúrgica durante una cirugía cardíaca por endocarditis infecciosa.

\section{Caso clínico}

Se trata de una paciente de sexo femenino, de 66 años, diabética, dislipidémica, hipertensa, hipotiroidea, que ingresó en la institución de los autores por un cuadro febril y episodio de hipotensión arterial.

Como antecedentes destacan que la paciente cursó internación, en otro centro, por infección urinaria causada por Enterobacter spp., por lo que cumplió tratamiento antibiótico dirigido. A su vez, un mes antes de la consulta recibió tratamiento odontológico (endodoncia). Su medicación habitual consistía en nevibolol, metformina y levotiroxina. 
A su ingreso se encontraba normotensa, con frecuencia cardíaca normal, afebril, con leves signos de congestión cardíaca. Se auscultó un soplo sistólico en foco mitral de moderada intensidad, irradiado a la axila izquierda. La radiografía de tórax mostró un índice cardiotorácico aumentado y signos de redistribución de flujo (Figura 1). El electrocardiograma evidenciaba trazado de ritmo sinusal con sobrecarga sistólica ventricular izquierda (Figura 2). En el laboratorio de ingreso se evidenciaba anemia y leucocitosis con predominio de segmentados. Tomándose hemocultivos que resultaron positivos para Streptococcus beta-hemolítico del grupo G, indicándose por ello tratamiento con vancomicina y, posteriormente, ampicilina.

Ante el posible diagnóstico de endocarditis infecciosa se decidió realizar una ecocardiografía transesofágica (ETE), donde se evidenció lo siguiente:

- Aurícula izquierda dilatada, ventrículo izquierdo de tamaño normal con fracción de eyección conservada (Vídeo 1), hipertrofia asimétrica a predominio septal $(20 \mathrm{~mm}$ ) que generaba obstrucción dinámica del tracto de salida izquierdo asociada a movimiento anterior sistólico (MAS) mitral (Vídeo 1, Vídeo 2 y Vídeo 5).

- Insuficiencia mitral severa por distintos mecanismos: MAS (Vídeo 2 y Vídeo 5), perforación de valva posterior a nivel del segmento P1 (Vídeo 2) (mecanismo Carpentier IB) con engrosamiento localizado en la superficie auricular del cuerpo de la valva posterior, en relación con el sitio de perforación, que podría corresponder a lesiones vegetantes antiguas; adicionalmente, otro jet de regurgitación central con dirección hacia la pared lateral de la aurícula por restricción de la valva posterior, que está engrosada y calcificada (Vídeo 3 a Vídeo 5) (mecanismo Carpentier III A).

- Válvula aórtica con cambios degenerativos y presencia de strands (Vídeo 1).

- Presión sistólica pulmonar normal (50 mmHg).

Una vez descartada la embolia periférica, se decidió realizar la cirugía cardíaca 4 semanas después del tratamiento antibiótico, realizándose reemplazo de válvula mitral con prótesis biológica N. ${ }^{\circ}$ 25, miomectomía septal y exclusión de la orejuela izquierda (doble sutura endocárdica) (Vídeo 6 y Vídeo 7). La prótesis mitral estaba normofuncionante con 2 pequeñas fugas periprotésicas sin repercusión hemodinámica.

En el postoperatorio mediato presentó FA, empiema por Enterobacter y septicemia por Staphylococus aureus resistente a meticilina, requiriendo pleurocentesis, decorticación pleural y tratamiento antibiótico con meropenem y vancomicina, con evolución satisfactoria.

Por persistencia del síndrome febril se realizó una nueva ETE en búsqueda de endocarditis protésica precoz (Vídeo 8 y Vídeo 9), sin nuevos hallazgos respecto a la prótesis mitral y con presencia de trombo en la OAl que se encontraba parcialmente comunicada con la aurícula izquierda, por una solución de continuidad mayor a $1 \mathrm{~cm}$.

\section{Discusión}

\section{Impacto clínico}

La FA es una complicación frecuente en el postoperatorio de cirugía cardíaca, observada hasta el $50 \%$ de los pacientes ${ }^{(2,4,5)}$. Diferentes estudios han observado que los episodios embólicos, tras la cirugía cardíaca, ocurren en pacientes que han presentado FA postoperatoria y que los trombos se originan predominantemente en la orejuela izquierda ${ }^{(2,6,7)}$. Teóricamente, la exclusión rutinaria de la OAl de la circulación sistémica, durante la realización de cirugía cardíaca, reduciría el riesgo de embolización. Ante esta evidencia se han desarrollado y empleado por más de 50 años diferentes técnicas de exclusión quirúrgica de la $\mathrm{OAl}$, a fin de reducir la probabilidad de ACV.

\section{Técnicas quirúrgicas}

Se han empleado técnicas endocárdicas y técnicas epicárdicas, de ligadura, resección, sutura endocárdica y resección grapada.

La mayoría de estos procedimientos se realizan concomitantemente durante la cirugía cardíaca abierta, pero también se pueden realizar epicárdicamente durante la ablación toracoscópica de FA o como procedimiento independiente puro, ya sea mínimamente invasivo (minitoracotomía) o toracoscópicamente.

Un estudio retrospectivo observacional, realizado por Ryam Kim et al., mostró una reducción significativa en la incidencia del ACV postoperatorio en aquellos pacientes que eran sometidos a ligadura de la OAl, durante todo tipo de cirugía cardíaca: valvular, cirugía de revascularización miocárdica, combinada y otras como el cierre de defecto septal y exéresis de mixoma ${ }^{(7)}$

Desafortunadamente, estudios retrospectivos, guiados por ETE, demostraron en el seguimiento que el cierre de la OAl es incompleto, con el riesgo potencial de que los trombos atraviesen esta comunicación y generen eventos embólicos posteriores. El criterio de cierre exitoso se estableció ante la presencia de un muñón menor a $1 \mathrm{~cm}$, y sin evidencia de flujo Doppler color en su interior.

En un trabajo retrospectivo, no aleatorizado en la Cleveland Clinic, realizado en 137 pacientes, que recibieron cierre quirúrgico de la OAl, Kanderian et al. presentaron la tasa de éxito de las diversas técnicas quirúrgicas. El resultado fue que sólo el 73\% de la resecciones, el 23\% de los cierres por sutura y el 0\% de los cierres por grapadora cumplieron el criterio de cierre exitoso ${ }^{(8)}$.

La oclusión incompleta tiene un impacto negativo en la salud de los pacientes en lo que respecta al seguimiento. En un trabajo presentado por García-Fernández et al. se evaluaron con ETE a 205 pacientes con ligadura de OAl en contexto de cirugía mitral. Después de un ajuste multivariado identificaron que el riesgo de ACV en los pacientes con cierre incompleto de OAl era mayor que en aquellos con orejuelas no intervenidas ${ }^{(9)}$.

En un estudio piloto prospectivo y aleatorizado de pocos pacientes, Lee $\mathrm{R}$ et al. compararon tres técnicas quirúrgicas diferentes: ligadura interna, escisión grapada y escisión quirúrgica, demostrando que ninguna técnica fue uniformemente exitosa a corto o largo plazo y la tasa de fracaso general fue del 57\%, concluyendo que la integridad de la OAI debe evaluarse por ETE intraoperatoria, de modo que ante la presencia de permeabilidad, se deben realizar procedimientos adicionales para lograr el éxito de la exclusión ${ }^{(10)}$.

El metanálisis desarrollado por Atti V. et al. (2018) resume los resultados de 12 estudios que compararon los eventos clínicos presentados en pacientes con FA preoperatoria, que recibieron tratamiento de exclusión quirúrgica de la OAI frente a aquellos en los que la OAI no fue intervenida, concluyendo que el cierre de la $\mathrm{OAl}$, realizado de forma concomitante durante la cirugía cardíaca, reduce la incidencia de ACV y eventos embólicos, sin aumentar el riesgo de mortalidad, de reoperación por sangrado, ni de FA postoperatoria. Las técnicas empleadas en estos ensayos fueron variables: doble sutura, amputación y resección grapada ${ }^{(11)}$. Los autores coinciden en que la interpretación de los resultados del metanálisis debe ser cuidadosa, debido a la insuficiente información clínica acerca del origen de los eventos isquémicos informados, y del protocolo de anticoagulación que recibieron los pacientes incluidos en los trabajos. A su vez, la mayoría de los estudios tienen un diseño retrospectivo o de bajo poder estadístico.

Existe un estudio en marcha, el Left Auricular Appendage Occlusion Study (LAAOS II), que presenta un diseño prospectivo, aleatorizado, doble ciego, multicéntrico e internacional. El objetivo de reclutamiento es de 4.700 pacientes y la hipótesis principal es que la oclusión de la OAI concomitante a la 
realización de cirugía cardíaca frente a la no oclusión de la OAl, en pacientes con FA prequirúrgica, reducirá el ACV o la embolia sistémica durante una media de seguimiento de 4 años, en ambos grupos con terapia anticoagulante adyuvante recomendada. Las técnicas quirúrgicas permitidas son: amputación y cierre con grapadora. Sin embargo, más estudios prospectivos y de mayor poder estadístico son necesarios para estandarizar protocolos de tratamiento.

\section{Recomendaciones de las guías}

Las guías europeas (ESC 2016) y americanas (AHA/ACC/HRS 2019) sobre e manejo de la FA, basándose en la evidencia publicada sobre las técnicas quirúrgicas tradicionales y el riesgo de eventos tromboembólicos, indican que el cierre quirúrgico de rutina de la OAl puede considerarse en pacientes con FA previa que son sometidos a cirugía cardíaca (indicación clase Ilb, nivel de evidencia B).

La ETE es la técnica de referencia diagnóstica para la detección de trombos en la OAI. Ante la falta de ensayos prospectivos a largo plazo sobre protocolos de anticoagulación en pacientes que han recibido el cierre quirúrgico de la OAl, las guías europeas recomiendan anticoagulación en todos los pacientes a pesar del cierre de OAl (clase I, nivel de evidencia B).

\section{Nuevas tecnologías}

Reconociendo las limitaciones del tratamiento quirúrgico tradicional, se han ido desarrollando dispositivos de cierre epicárdicos para lograr la exclusión completa y permanente de la OAI con un rango de mayor seguridad.

El sistema de cierre epicárdico de la OAl por clip (AtriClip ${ }^{\circledR}$ ) consiste en un dis positivo implantable, a base de nitinol, de cierre automático, con una herramienta de despliegue reutilizable. Existen diferentes tipos de dispositivos de cierre (Figura 3).

Salzberg et al., en 2010, demostraron que la oclusión con clip epicárdico es un procedimiento seguro y efectivo para el cierre de la orejuela izquierda en una población sometida a cirugía cardíaca ${ }^{(12)}$. En este ensayo no se presentaron complicaciones relacionadas al procedimiento y todos los dispositivos fueron evaluados por ETE intraoperatoria y por tomografía computarizada a 3 meses para confirmar el éxito. Se obtuvieron resultados similares en un ensayo multicéntrico, prospectivo, realizado por Ailawadi et al. en $2011^{(13)}$.

Caliskan et al. publicaron en 2018 el primer trabajo a largo plazo que demos tró, en una población de 291 pacientes sometida a cirugía cardíaca, que el cierre de la OAI mediante implante de AtriClip ${ }^{\circledR}$ fue seguro, eficaz y durable en un seguimiento mayor a 5 años. Basados en un riesgo esperado de ACV observaron una reducción significativa de los eventos cerebrales en un seguimiento de 3 años. Se trató de un estudio no aleatorizado, que permitió realizar un seguimiento por tomografía del dispositivo. Se observó una reducción de la tasa de ACV, en comparación a la tasa esperable, en un grupo de pacientes con similar puntuación de $\mathrm{CHA}_{2} \mathrm{DS}_{2}$-VASc (tasa esperada de 4,0/100 pacientes/año) ${ }^{(14)}$. Hasta el momento se han implantado en el mundo más de 100.000 dispositivos AtriClip ${ }^{\oplus}$, sin documentarse complicaciones cardíacas mayores.

\section{Panorama en presencia de ritmo sinusal}

No existe evidencia del cierre quirúrgico de la OAl en pacientes sin antecedentes de FA prequirúrgica.

Actualmente se encuentra en marcha un estudio para evaluar la reducción del ACV en pacientes con ritmo sinusal preoperatorio, el estudio AtriClip Left
Atrial Appendage exclusión Concomitant to Structural Heart Procedures (ATLAS), que consiste en un ensayo prospectivo aleatorizado, multicéntrico, que busca comparar el impacto de la FA postoperatoria en pacientes con ritmo sinusal prequirúrgico. Se definieron dos ramas de tratamiento: pacientes con FA postoperatoria y cierre quirúrgico de OAI mediante dispositivo epicárdico AtriClip ${ }^{\circledast}$ frente a pacientes con FA postoperatoria sin cierre quirúrgico de OAl. Se estima el reclutamiento en 2.000 pacientes y los resultados serán de vital importancia en la toma de decisiones clínicas.

\section{Conclusiones}

La OAl es la fuente embolígena más frecuente relacionada con ACV y FA. Si bien la evidencia publicada está sujeta a sesgos de investigación por la formulación de los trabajos publicados, la exclusión quirúrgica de la OAl es una estrategia segura para la prevención de ACV relacionado con la presencia de trombos en pacientes que reciben tratamiento quirúrgico de patología cardíaca y FA preoperatoria.

La exclusión de OAI mediante técnicas tradicionales, como la sutura, ligadura, resección o escisión por grapadora, pueden resultar en un cierre incompleto de la misma.

Las experiencias realizadas con dispositivos de cierre epicárdico señalan resultados prometedores en relación a seguridad y durabilidad del procedimiento. No obstante, no se conoce la evolución a largo plazo en muestras de mayor número de pacientes.

La ETE intraoperatoria es un método altamente sensible y disponible para detectar trombos y definir el éxito de la exclusión de la OAI.

Finalmente, se requieren trabajos aleatorizados que comparen la exclusión de la orejuela con y sin tratamiento anticoagulante concomitante para indicar la suspensión de la anticoagulación tras la exclusión de la OAI.

\section{Estudio por imagen}

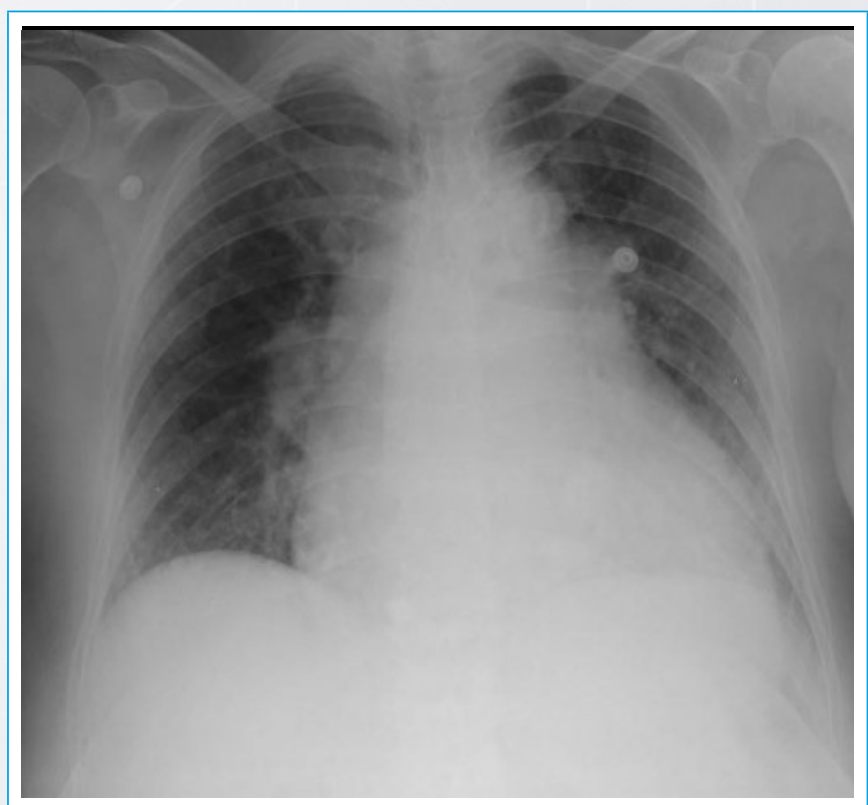

Figura 1. Radiografía de tórax de frente de ingreso 


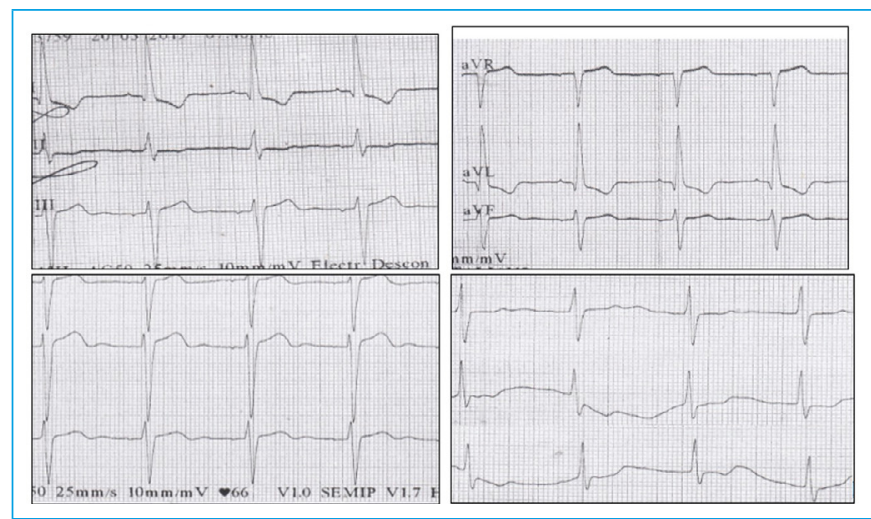

Figura 2. Electrocardiograma de ingreso

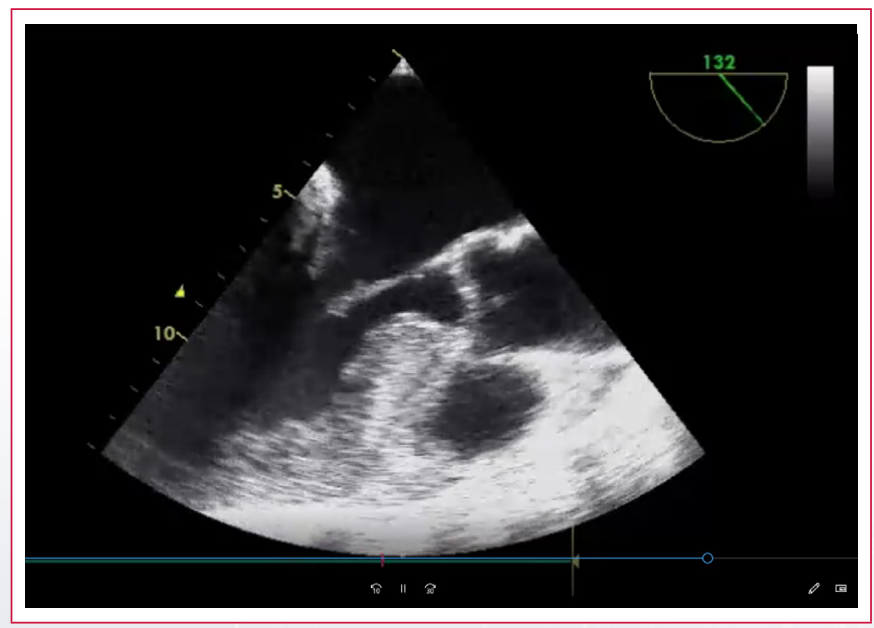

Vídeo 1. Ecocardiografía transesofágica, en esófago medio a $132^{\circ}$, donde se evidencia dilatación auricular izquierda, ventrículo izquierdo de tamaño normal con fracción de eyección conservada, hipertrofia asimétrica a predominio septal $(20 \mathrm{~mm})$ y movimiento anterior sistólico mitral que ocasiona obstrucción dinámica del tracto de salida izquierdo. Adicionalmente, cambios degenerativos en válvula aórtica, engrosamiento y retracción de valva posterior mitral con engrosamiento en la superficie auricular de la misma, que podría tratarse de vegetaciones antiguas

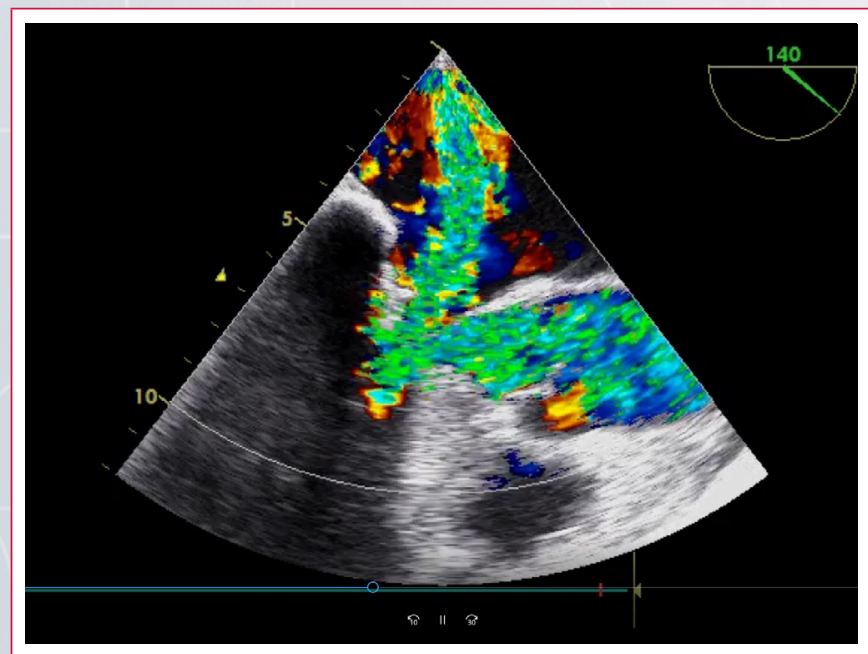

Vídeo 2. Ecocardiografía transesofágica, en esófago medio a $140^{\circ}$ con color, donde se evidencia obstrucción dinámica del tracto de salida izquierdo y doble jet de insuficiencia mitral

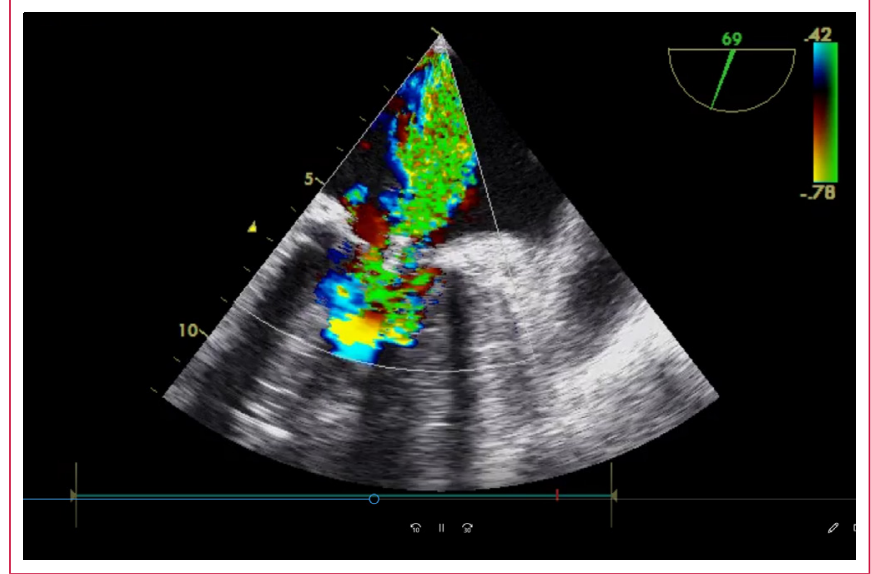

Vídeo 3. Ecocardiografía transesofágica, en esófago medio a $69^{\circ}$ con color con ligera rotación desde la toma anterior, donde se evidencia mejor el jet central de insuficiencia mitral

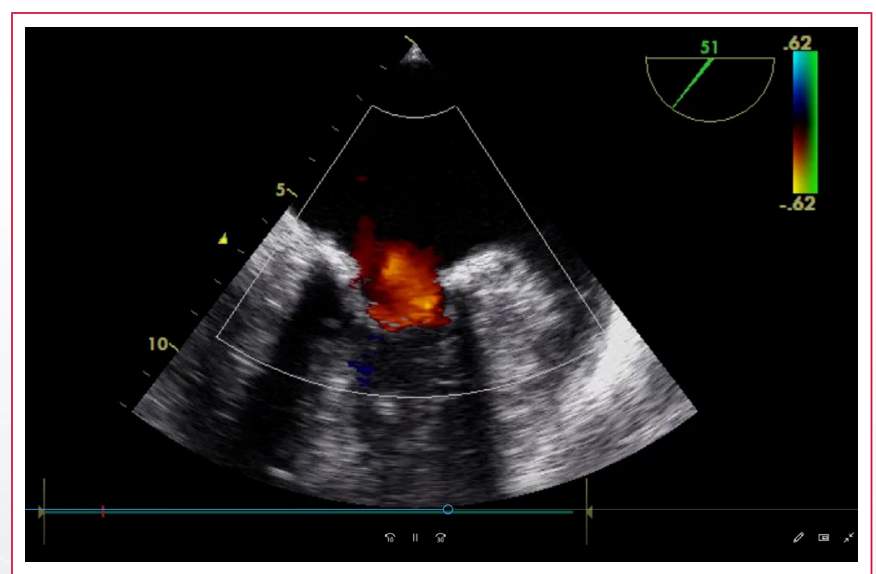

Vídeo 4. Ecocardiografía transesofágica, en esófago medio a $51^{\circ}$ con color, con ligera rotación desde la toma anterior, donde se evidencia mejor el jet central de insuficiencia mitral

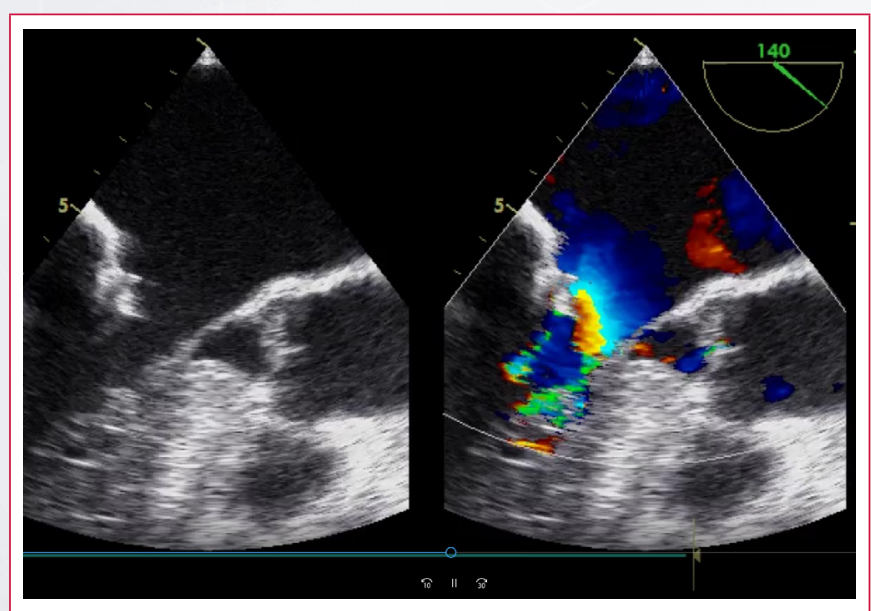

Vídeo 5. Ecocardiografía transesofágica, en esófago medio a $140^{\circ}$, con y sin color, donde se evidencia dilatación auricular izquierda, ventrículo izquierdo de tamaño con fracción de eyección conservada, hipertrofia asimétrica a predominio septal $(20 \mathrm{~mm})$, movimiento anterior sistólico mitral con obstrucción dinámica del tracto de salida izquierdo, cambios degenerativos en válvula aórtica, engrosamiento y retracción de valva posterior mitral con engrosamiento en la superficie auricular de la misma y jet severo de insuficiencia mitral 


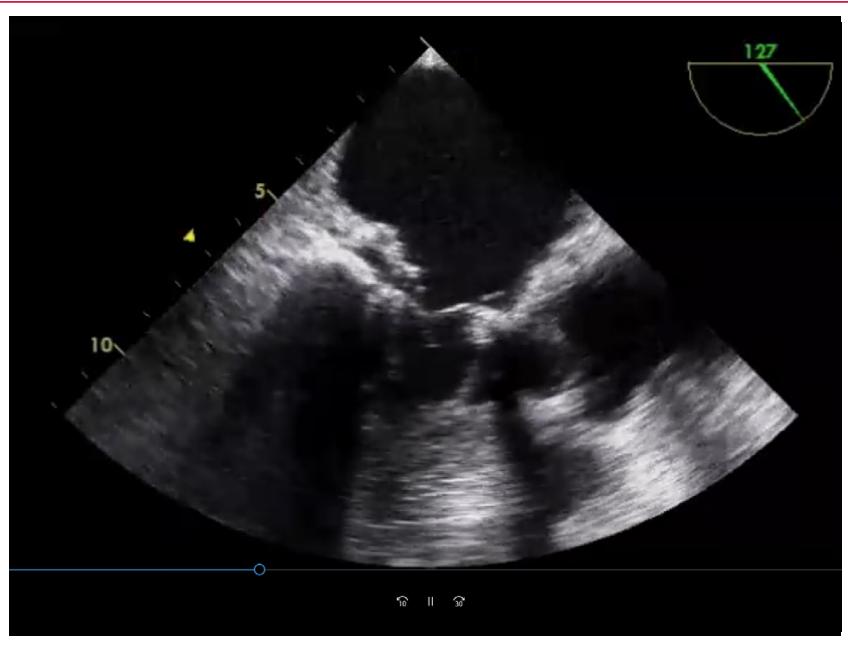

Vídeo 6. Ecocardiografía transesofágica postoperatoria, en esófago medio a $127^{\circ}$, donde se evidencia prótesis biológica $N .^{\circ} 25$ normofuncionante y disminución del diámetro del septum anterior basal por miomectomía septal

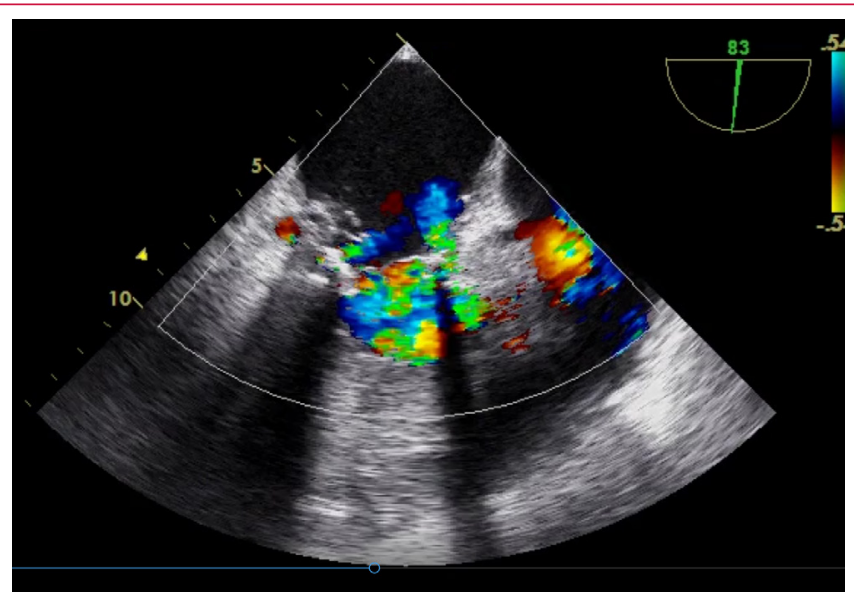

Vídeo 7. Ecocardiografía transesofágica postoperatoria, en esófago medio a $83^{\circ}$, con color. La prótesis mitral estaba normofuncionante con 2 pequeñas fugas periprotésicas sin repercusión hemodinámica

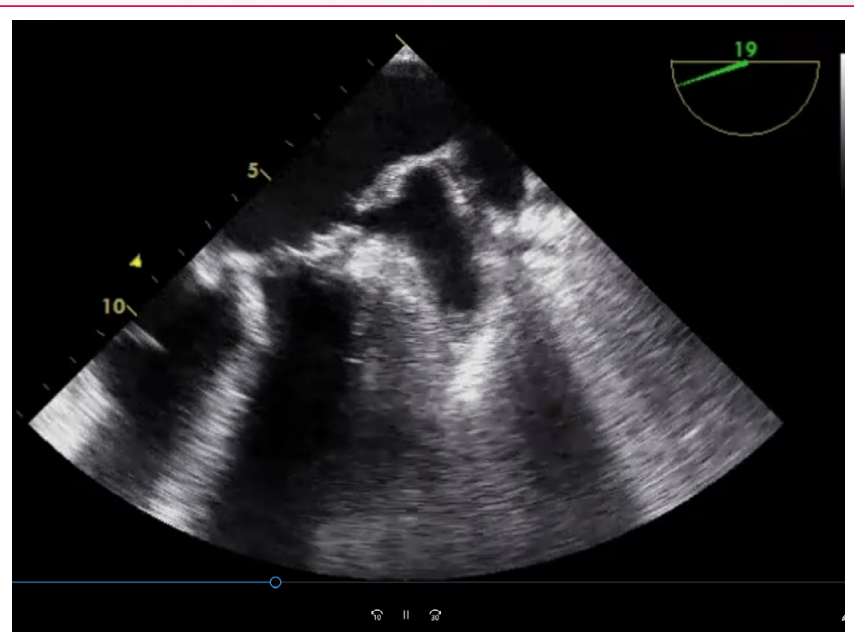

Vídeo 8. Ecocardiografía transesofágica postoperatoria, en esófago medio a $19^{\circ}$, enfocada en orejuela izquierda, evidenciándose por una solución de continuidad mayor a $1 \mathrm{~cm}$ con la aurícula izquierda

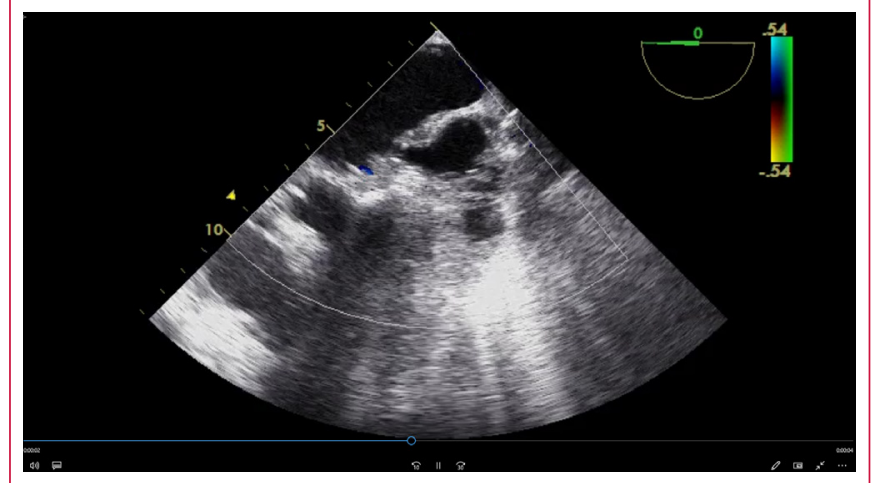

Vídeo 9. Ecocardiografía transesofágica postoperatorio, en esófago medio a $19^{\circ} \mathrm{con}$ color, enfocada en orejuela izquierda, evidenciándose además de solución de continuidad con la aurícula izquierda, presencia de trombo en la OAl

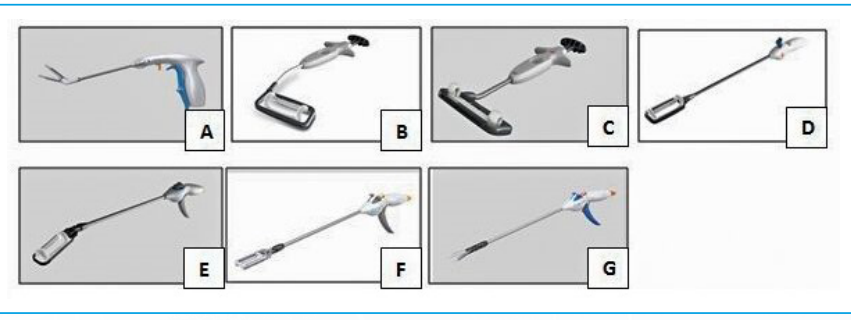

Figura 3. Dispositivos de cierre de orejuela izquierda. A: AtriClip ${ }^{\oplus}$ FLEX $V_{\text {; }}$ B: AtriClip ${ }^{\oplus}$ FLEX; C: AtriClip ${ }^{\oplus}$ Standard; D: AtriClip ${ }^{\oplus}$ Long; E: AtriClip ${ }^{\oplus}$ PRO; F: AtriClip ${ }^{\oplus R O} 2$

\section{Abreviaturas}

- OAl: orejuela auricular izquierda

- FA: fibrilación auricular

- ACV: accidente cerebrovascular

- AlT: accidente isquémico transitorio

- ETE: ecocardiografía transesofágica

- TC: tomografía computarizada

\section{Ideas para recordar}

La evidencia publicada sobre las diferentes técnicas quirúrgicas demostró que el cierre de la orejuela izquierda es un procedimiento seguro.

- Con tasas de éxito variable según la técnica empleada, el cierre efectivo de la orejuela izquierda reduce el riesgo de ACV en el postoperatorio de cirugía cardíaca y FA preoperatoria.

- La ecocardiografía transesofágica debe indicarse en toda cirugía cardíaca donde se realice la oclusión de la OAI para definir el éxito del procedimiento.

- Estudios aleatorizados, prospectivos y de mayor poder estadístico se encuentran en marcha, que servirán para protocolizar la práctica clínica y quirúrgica.

\section{Bibliografía}

1. Chugh SS, Havmoeller R, Narayanan K, et al. Worldwide epidemiology of atrial fibrillation: a Global Burden of Disease 2010 study. Circulation 2014; 129: 837-847.

2. Blackshear JL, Odell JA. Appendage obliteration to reduce stroke in cardiac surgical patients with atrial fibrillation. Ann Thorac Surg 1996; 61: 755-759. 
3. Wang TJ, Massaro JM, Levy D, et al. A risk score for predicting stroke or death in individuals with new-onset atrial fibrillation in the community: the Framingham Heart Study. JAMA 2003; 290: 1049-1056.

4. Auer J, Weber T, Berent R, et al. Risk factors of postoperative atrial fibrillation after cardiac surgery. J Card Surg 2005; 20: 425-431.

5. Johnson WD, Ganjoo AK, Stone CD, et al. The left atrial appendage: our most lethal human attachment! Surgical implications. Eur J Cardiothorac Surg 2000; 17: 718-722.

6. Syed TM, Halperin JL. Left atrial appendage closure for stroke prevention in atrial fibrillation: state of the art and current challenges. Nat Clin Pract Cardiovasc Med 2007; 4: 428-435.

7. Kim R, Baumgartner N, Clements J. Routine left atrial appendage ligation during cardiac surgery may prevent postoperative atrial fibrillation-related cerebrovascular accident. J Thorac Cardiovasc Surg 2013; 145: 582-589.

8. Kanderian AS, Gillinov AM, Pettersson GB, et al. Success of surgical left atrial appendage closure: assessment by transesophageal echocardiography. $J$ Am Coll Cardiol 2008; 52: 924-929.

9. García-Fernández MA, Pérez-David E, Quiles J, et al. Role of left atrial appendage obliteration in stroke reduction in patients with mitral valve prosthe- sis: a transesophageal echocardiographic study. J Am Coll Cardiol 2003; 42 1253-1258

10. Lee R, Vassallo P, Kruse J, et al. A randomized, prospective pilot comparison of 3 atrial appendage elimination techniques: Internal ligation, stapled excision, and surgical excision. J Thorac Cardiovasc Surg 2016; 152 (4): $1075-$ 1080.

11. Atti V, Anantha-Narayanan M, Turagam M, et al. Surgical left atrial appendage occlusion during cardiac surgery: A systematic review and meta-analysis. J Cardiol 2018; 10 (11): 242-249.

12. Salzberg SP, Plass A, Emmert MY, et al. Left atrial appendage clip occlusion: early clinical results. J Thorac Cardiovasc Surg 2010; 139: 1269-1274.

13. Ailawadi G, Gerdisch MW, Harvey RL, et al. Exclusion of the left atrial appendage with a novel device: early results of a multicenter trial. J Thorac Cardiovasc Surg 2011; 142: 1002-1009.e1.

14. Caliskan E, Sahin A, Yilmaz M, et al. Epicardial left atrial appendage AtriClip occlusion reduces the incidence of stroke in patients with atrial fibrillation undergoing cardiac surgery. Europace 2018; 20 (7): e105e114. 